\title{
Targeted IL-12 resets the immune response to gonorrhoea
}

Researchers at the University of Buffalo, SUNY have taken the first steps towards resetting the immune response to gonorrhoea, with a sustained-release cytokine treatment that promotes clearance of the infection and the development of prolonged resistance to reinfection in mice.

The sexually transmitted disease gonorrhoea is an urgent public health threat because of resistance to antibiotic treatment. Gonorrhoea also has resistance to the human immune system, which is demonstrated by high reinfection rates.

\section{1 ...converts the infection into} a live vaccine... 77

Prospects have looked bleak in the fight against gonorrhoea. Worldwide, more than 100 million gonococcal infections are estimated to occur each year. No vaccine is available, and antibiotic treatment options are increasingly limited. The new research shows that great potential to combat gonorrhoea already exists in the immune system.
The SUNY researchers, led by Michael Russell, hypothesized that Neisseria gonorrhoeae diverts the host's defences away from an adaptive immune response and towards the innate system, which it is able to tolerate. They sought to reverse this response with proinflammatory cytokine treatment, and were aided by the felicitous development of microencapsulated interleukin 12 (IL-12), enabling vaginal application of the slow-release IL-12 formulation in a mouse model of gonorrhoea infection.

As hoped, an adaptive immune response developed, with production of specific antibodies and substantially quicker clearance of infection compared with untreated mice. Furthermore, mice treated with IL-12 during initial infection retained a level of immunity to gonorrhoea. A subsequent bacterial challenge, without further IL-12 treatment, was met by renewed antibody production and enhanced clearance. According to Russell, this immune memory persists at least 6 months after the initial challenge.
Microencapsulated neutralizing antibodies to TGF- $\beta$ and IL-10 had similar effects, supporting a theory that these cytokines are induced during N. gonorrhoeae infection.

This low-dose, sustained-release delivery system avoids toxicity associated with cytokine treatment. Potential effects on reproductive function have yet to be determined, but might be outweighed by the serious risks accompanying an untreated infection.

If, as Russell believes, this treatment "converts the infection into a live vaccine," it could be effective against other diseases, and it might be a valuable approach to reducing dependence on a dwindling arsenal of antibiotics.

Robert Phillips

Original article Liu, Y. et al. Enhancement of adaptive immunity to Neisseria gonorrhoeae by local intravaginal administration of microencapsulated interleukin 12. J. Infect. Dis. doi:10.1093/infdis/jit354 